\title{
Controvérsias na cirurgia da origem anômala das artérias coronárias
}

\author{
E. Régis JUCÁ*
}

RBCCV 44205-300

\begin{abstract}
Jucá E R - Controvérsias na cirurgia da origem anômala das artérias coronárias. • Rev Bras Cir Cardiovasc 1996; $11(2): 126-8$.

RESUMO: Seis casos de origem anômala das artérias coronárias são relatados: artéria coronária esquerda nascendo no tronco pulmonar 3 casos, artéria coronária direita nascendo no tronco pulmonar $2 \mathrm{e}$ uma coronária direita nascendo no seio de Valsalva esquerdo. Foi realizado a reparo cirúrgico sem mortalidade. As controvérsias sobre as opçōes cirúrgicas são discutidas.
\end{abstract}

DESCRITORES: Anomalias dos vasos coronários, cirurgia.

\section{INTRODUÇÃO}

As anomalias congênitas das artérias coronárias podem ser classificadas como anomalias na origem, no trajeto e na terminação (fístulas) (1).

No presente trabalho analisaremos 6 casos de anomalia da origem, já que a nossa experiência com as fístulas coronárias já foi anteriormente publicada.

Inúmeros procedimentos foram e continuam sendo propostos para a origem anômala das artérias coronárias $(\mathrm{OACO})$, sendo que a mais desejável é o reimplante, segundo a literatura recente.

A mortalidade no reparo da OACO varia de $0 \%$ * a $31 \%{ }^{(2)}$, razão pela qual pensamos que a conduta de cada caso deve ser individualizada.

\section{CASUISTICA E MÉTODOS}

Foram operados pelo autor, no Hospital de Messejana e na Casa de Saúde São Raimundo, 6 pacientes de OACO assim distribuídos quanto à origem:

Grupo 1 - Coronária esquerda no tronco pulmonar 3

Grupo 2 - Coronária direita no tronco pulmonar 2

Grupo 3 - Coronária direita no seio de Valsalva esquerdo 1

Os 3 pacientes do Grupo 1 tinham entre 3 meses e 2 anos de idade e a operação consistiu na ligadura do tronco de coronária esquerda, já que, apesar da cardiomegalia e quadro de miocardiopatia, havia extensa rede de colaterais.

No grupo 2, 1 paciente de 17 anos tinha uma comunicação interventricular (CIV) associada a origem anômala da coronária direita (CD) no tronco pulmonar. A operação consistiu no reimplante da CD na aorta e fechamento da CIV via átrio direito. O outro paciente tinha 42 anos e era portador de estenose aórtica calcificada. A operação consistiu

\footnotetext{
Trabalho realizado nos Serviços de Cirurgia Cardiovascular da Casa de Saúde São Raimundo e Hospital de Messejana. Fortaleza, CE, Brasil. Apresentado ao $23^{\circ}$ Congresso Nacional de Cirurgia Cardiaca. Recife, PE, 20 a 23 de março, 1996.

* Da Casa de Saúde São Raimundo e do Hospital de Messejana. Fortaleza.

Endereço para correspondência: E. Régis Jucá. Rua Manuel Jesuino, 50/1401. CEP: 61025-270, Fortaleza, CE, Brasil. Tel. [085] 224.4663.
} 
em implante de uma prótese aórtica metálica e ligadura da origem da CD.

A paciente do Grupo 3 que apresentava angina do peito por compressão da CD, em virtude da origem no seio de Valsalva esquerdo foi submetida a implante de ponte de safena para coronária direita.

\section{RESULTADOS}

Não houve mortalidade nem complicações cirúrgicas maiores. No Grupo 1 todos os pacientes melhoraram os índices do ecocardiograma. O ECG melhorou e a cardiomegalia desapareceu, com normalização do índice cardiotorácico. Os 3 encontram-se em classe I da N Y H A, com execelente evolução clínica.

A paciente com CIV associada à origem anômala da $C D$ apresentou sangramento excessivo pelo dreno torácico e foi reoperada na noite da operação. Sua evolução tardia é excelente, tendo sido operada para correção de hérnia incisional logo abaixo do esterno.

O paciente que recebeu uma prótese aórtica apresenta-se em classe I da $\mathrm{N} \mathrm{Y} \mathrm{H} \mathrm{A,} \mathrm{em} \mathrm{regime}$ de anticoagulante oral. $O$ teste ergométrico pósoperatório foi negativo, apresentando ECG normal e ausência de dor.

A paciente com origem da CD no seio de Valsalva esquerdo apresentou dor atípica no pósoperatório tardio e foi submetida a nova cinecoronariografia, que revelou ponte de safena pérvia com excelente fluxo e oclusão da.CD na origem, na zona de compressão, Evolução satisfatória.

\section{COMENTÁRIOS}

Inúmeras operações têm sido propostas para reparo da origem anômala da artéria coronária esquerda (CE) na pulmonar: ligadura ${ }^{(3)}$, anastomose subclávia-coronária, túnel intrapulmonar (Takeuchi), ponte venosa ou arterial, e reimplante com hipotermia profunda e parada circulatória $(2,4,5)$. A mortalidade varia de $0 \%$ observada por TURLEY* a $31 \%$ demonstrada por VOHUE et al. (2) em pacientes com fração de ejeção abaixo de $20 \%$ indo a $75 \%$, segundo ARDEHALI et al. (4). A nossa observação em Serviços visitados é que o número de complicações não é pequeno, exigindo a assistência cir-

* TURLEY K - Anomalous origin of left coronary artery. Apresentado ao $27^{\circ}$ Annual Meeting of the Society of Thoracic Surgeons. Palm Springs, Janeiro 1995. culatória mecânica, o transplante ou, mesmo, levando ao óbito. BACKER et al. (5) relataram 1 caso de transplante cardíaco após tentativa de reimplante.

Como os nossos casos apresentavam baixa fração de ejeção, optamos pela ligadura do tronco da CE com toracotomia esquerda, aguardando os resultados para uma $2^{\mathrm{a}}$ operação imediata ou após algum tempo.

SABISTON (em comunicação pessoal), com toda a força da Duke Database, nos afirmou que muitos pacientes com cirurgia para construção do duplo sistema melhoraram apesar de funcionar só o sistema direito.

Como sabemos, estes pacientes apresentam uma extensa circulação colateral. Nossos 3 pacientes sobreviveram em função desta extensa rede de colaterais. Com a função de VE melhorada, deverão ser submetidos a testes de esforço ou estudos com thalio para indicação de uma $2^{a}$ intervenção, se necessário.

Tal conduta é passível de críticas, mas, como a mortalidade é alta com assistência circulatória mecânica em crianças e, como não dispomos do instrumental necessário, optamos por uma conduta mais conservadora $(2,5)$.

A origem anômala da CD no tronco pulmonar é mais rara e mais benigna. Nossos 2 pacientes passaram da adolescência e ambos apresentavam lesão associada: CIV em uma e extenose aórtica calcificada por valva bivalvulada que recebeu prótese aórtica em outro. FERNANDES et al. (1) e COOLEY et al. (6) encontraram, entre 1964 a 1989, 58 casos de origem da coronária esquerda no tronco pulmonar e 2 da coronária direita no tronco pulmonar.

Nos nossos 2 casos optamos pelo reimplante em 1 e ligadura em outro. O teste ergométrico no paciente que recebeu prótese aórtica e ligadura na origem da CD em tronco pulmonar foi negativo, demonstrando que a exuberante circulação colateral vista anatomicamente era fisiologicamente efetiva. A origem da coronária $D$ na aorta no seio de Valsalva esquerdo é lesão rara, que tem recebido atenção especial graças aos trabalhos de ROBERTS (7), demonstrando a possibilidade de morte súbita. Existem inúmeras opções cirúrgicas e nós escolhemos a realização de ponte de safena, por acreditar que a safena tem um bom run-off e, desde que o paciente não apresente distúrbios do metabolismo lipídico, a obstrução deve ser rara.

Embora a nossa estatística seja reduzida, a ausência de mortalidade e os bons resultados tardios nos permitem afirmar que a conduta cirúrgica deve ser individualizada. 
Do ponto de vista do diagnóstico, inferimos que - ECO é valioso instrumento no diagnóstico da OACO, tanto para CD como para CE. Toda criança com quadro de miocardiopatia dilatada deve ter um ECO para o diagnóstico diferencial com OACO.

Por outro lado, a angina em pacientes jovens, na ausência de outros sintomas, pode chamar a atenção para origem anômala da coronária $D$ no seio de Valsalva esquerdo, que é uma causa de morte súbita em adultos jovens $(7,8)$.

Desde que BLAND et al. (9), do Massachusetts General Hospital descreveram, em 1933, a origem anômala da coronária no tronco pulmonar, muitos progressos foram conquistados, porém controvérsias persistem neste discutido setor da má-formação congênita das coronárias.

RBCCV 44205-300

Jucá E R - Controversies on the surgical options for anomalous origin of the coronary arteries. Rev Bras

Cir Cardiovasc 1996; 11 (2): 126-8.

ABSTRACT: Six cases of anomalous origin of the coronary arteries are reported: left arising from pulmonary artery $(n=3)$, right arising from pulmonary artery $(n=2)$, and 1 case of right coronary with origin in the left sinus of Valsalva. They were submited to surgical repair without mortality. The controversies on the surgical options are discussed.

DESCRIPTORS: Coronary vessels anomalies, surgery.

\section{REFERÊNCIAS BIBLIOGRÁFICAS}

1 Fernandes E D, Kadivar H, Hallman G L, Reul G, Ott $D$, Cooley D A - Congenital malformations of the coronary arteries: the Texas Heart Institute experience. Ann Thorac Surg 1992; 54: 732-40.

2 Vouhê P R, Tamisier D, Sidi D - Anomalous left coronary artery from the pulmonary artery: results of isolated aortic reimplantation. Ann Thorac Surg 1992; 54: $621-6$.

3 Sabiston, D C, Neil C A, Taussig, H B - The direction of blood flow in anomalous lest coronary artery arising from the pulmonar artery. Circulation 1960; 22: 5917.

4 Aardehali A, Laks H, Aallada V. - Management of the anomalous origin of the left coronary artery from the pulmonary artery. In: Advances in cardiac surgery. New York: C. V. Mosby Company, 1996.
5 Backer C L, Stout M J, Muster A, Idriss F, Mavroudis CM - Anomalous origin of the left coronary artery. $J$ Thorac Cardiovasc Surg 1992; 103: 1049-55.

6 Cooley D A, Hallman, G L, Bloodwell R D - Definitive qualified treatment of anomalous origin of left coronary artery from pulmonary artery. J Thorac Cardiovasc Surg 1966; 52: 789-808.

7 Roberts W C - Major anormalies of coronary arterial origin seen in adult mood. Am Heart $J$ 1986; 111: 941-63.

8 Rinaldi R G, Carballido J, Giles R, Tord E D, Porro R - Right coronary artery with anomalous origin. Ann Thorac Surg 1994; 58: 828-34.

9 Bland E F, White P D, Garland J - Congenital anomalies of the coronary arteries: report of an unusual case. Am Heart $J$ 1933; 9: 787-801. 